\title{
SALUD
}

\section{Microaspiradora para la extracción de tumores cerebrales, con microdestrucción y sistema de control integrado}

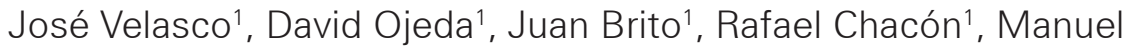 \\ Benítez ${ }^{1}$, Rebeca Meléndez ${ }^{1}$
}

\section{Resumen}

Introducción: El propósito fundamental de ésta investigación tuvo como finalidad diseñar una microaspiradora para la extracción de tumores cerebrales con seccionadores de cánulas de aspiración con microdestrucción y sistema de control integrado. Con el desarrollo del proyecto se pretende extraer la mayor cantidad de tumor cerebral y de ésta manera mejorar el nivel de vida del paciente. Para esto se desarrolló el diseño de diferentes tipos de herramientas de corte, obteniéndose así un seccionador para tejidos duros y otro para tejidos blandos. Por otro lado, se diseñó mediante el uso de las técnicas de vacío y mecánica de los fluidos, cánulas de aspiración las cuales constan de una pequeña boquilla metálica que estará en contacto directo con el paciente. Luego, se diseñó un sistema de control de lazo abierto que monitoreará y controlará las variables del sistema de succión, bombeo de líquido de irrigación y control de nivel en los depósitos de materia tumoral, con la finalidad de obtener un equipo de fácil uso. Posteriormente se verificó por medio del simulador Automation Studio 3.0.5, el funcionamiento del sistema de control y finalmente se realizó un análisis de factibilidad técnica y económica obteniéndose que la solución definitiva es viable para su implementación en el país.

Objetivo: Diseño de una microaspiradora para la extracción de tumores cerebrales con seccionadores de cánulas de aspiración con microdestrucción

Material y Método: La investigación se considera de nivel exploratoria, puesto que existe escaso acceso a información, diseños y funcionalidad, referente a sistemas de control para equipos de succión de tipo quirúrgico o similares utilizados, existentes en el mercado nacional e internacional, esto debido a que los fabricantes de dichos equipos se reservan datos técnicos referentes a su diseño, sin embargo algunas características, como las de instalación y uso, poseen accesibilidad. Es una investigación factible, debido a que se plantea una propuesta viable que satisface las necesidades establecidas en los objetivos y alcance, además tuvo apoyo en investigaciones previas como Ojeda et al.

1 Universidad de Carabobo, Venezuela.

Fue publicado en la Revista de Ingeniería de la Universidad de Carabobo.

E-mail: ingjosevelasco@gmail.com

DOI: 10.26885/rcei.foro.2018.124 


\section{Microaspiradora para la extracción de tumores cerebrales. Velasco et al.}

(2007) y Velasco et al. (2014), siendo así una investigación de tipo documental. Resultados: Se logró el diseño de dos clases de seccionadores (tipo fresas odontológicas), uno para tumores duros (huesos, tejidos calcificados, etc.) con forma en la parte activa cilíndrica, hemisférica; y otro para tejidos blandos (como por ejemplo tejidos de consistencias similares al hígado, riñón, cartílago, piel.) con forma en la cabeza cilíndrica punta plana.

Conclusiones: Se logró considerable mejoras, en comparación a la propuesta de Ojeda at al (2007) y Velasco et al. (2014), son la longitud de cánula de tres (3) metros mayor a la propuesta por ellos, agrupando la automatización del equipo, selección del depósito para restos de tumor y sangre, la mejora en la implementación de un sistema de alimentación de líquido de irrigación y la inclusión de los seccionadores en la cánulas de succión con un sistema optimo y silencioso para la micro-destrucción de tejidos tumorales bien sean tumores duros o blandos.

Palabras clave: Microaspirador, microdestrucción, boquillas, quirúrgico, cirugía, tumores.

\section{Referencias}

Atkinson, L. J. \& Nancymarie, H. F. (1998). Técnicas de quirófano. Madrid, España: Harcourt Brace.

Fuller, J. K. (2007). Instrumentación quirúrgica. Teoría, técnicas y procedimientos. Medica Panamericana: Editorial, México.

Ojeda, D., Gámez, B., Velasco, J., Chacón, R. (2007). Diseño de una micro aspiradora para extraer tumores cerebrales. Revista Ingeniería UC, 14(3), 24-29.

Velasco, J., Ojeda, D., Hurtado, M., Chacón, R. (2014). Diseño de un seccionador con cánula de aspiración para una microaspiradora de extracción de tumores cerebrales. Revista Ingeniería UC, 21(3), 65-75. 